\title{
A New Doubly Salient Permanent Magnet Motor
}

\author{
Ming Cheng, K.T. Chau and C.C. Chan \\ Department of Electrical and Electronic Engineering \\ The University of Hong Kong \\ Pokfulam Road, Hong Kong
}

\begin{abstract}
In this paper, a new 8/6-pole doubly salient permanent magnet (DSPM) motor is proposed and analyzed. The key is to analytically derive the output power density of the DSPM motor in terms of the rotor-to-stator pole ratio. Hence, the analytical design, finite element analysis and computer simulation of the proposed 8/6. pole DSPM motor are presented. The results show that this DSPM motor has following major features: it can achieve higher power density than an induction motor; its power density is proportional to the ratio of rotor pole and stator pole numbers; there is a maximum speed limitation which is proportional to the voltage but inversely proportional to the phase winding turns and the rate of change of PM flux; at high speed, the distribution of phase current is asymmetric and current regulation is needed in the negative stroke. Compared with the well known 6/4pole DSPM motor, the proposed 8/6-pole one offers higher power density, wider speed range and less phase current magnitude.
\end{abstract}

\section{Introduction}

Starting from the past decade, the switched reluctance' (SR) motor has received much research efforts. It offers the advantages of simple structure, fault tolerance and mechanical robustness, leading to be attractive for industrial and electric vehicle (EV) applications [1]. Nevertheless, its inherent limitations in efficiency and power density as compared to the permanent magnet brushless dc motor [2] have prompted the research into the incorporation of permanent magnets into the basic SR motor structure. Recently, a viable topology, named as the doubly salient permanent magnet (DSPM) motor, has been introduced [3], [4]. This DSPM motor essentially adopts the same structure as a SRM but with permanent magnets placed in the stator or rotor. Recent literatures have illustrated that the DSPM motor is of high efficiency, high power density and simple structure.

Similar to the SR motor, the DSPM motor may be of the combination of 6 stator poles and 4 rotor poles (so-called the 6/4-pole), the 8/6-pole, or other pole combinations that can accord to its basic operation principle. To the best of the authors' knowledge, the available publications of the DSPM motor have been dealt with the 6/4pole combination only [3]-[8]. Possible reasons may be due to the impression that the 6/4-pole motor is simpler than the 8/6-pole one in terms of both construction and control. It is the purpose of this paper to reveal the fact that the 8/6-pole DSPM motor has its definite advantages over the 6/4-pole one, though it is slightly more complicated in construction and control.

\section{Steady-State Analysis}

Fig.1 shows the cross section of the proposed 4phase 8/6-pole DSPM motor. The corresponding theoretical waveforms of PM flux $\phi_{m}$ and phase current $i$ are shown in Fig. 2. Since the applied voltage $U$ is the phase voltage $u$ of each phase winding, the per-phase input power $P$ can be expressed as:

$P=\frac{1}{T} \int_{0}^{T} u i d t=\frac{1}{T}\left[\int_{t_{1}}^{t_{2}} U I_{m} d t+\int_{t_{3}}^{t_{4}}(-U)\left(-I_{m}\right) d t\right]$ $=\frac{1}{T} 2 U I_{m} \Delta T$

where $T=\frac{\tau_{r}}{\omega_{r}}, \Delta T=\frac{\theta_{w}}{\omega_{r}}, \tau_{r}=\frac{2 \pi}{p_{r}}$ is the rotor pole pitch, $\omega_{r}$ is the angular velocity of the motor and time $t_{1} \sim t_{4}$ are the corresponding rotor positions of $\theta_{1} \sim \theta_{4}$. So, (1) can also be expressed as:

$P=2 U I_{m} \frac{\theta_{w}}{\tau_{r}}$

For the motor with $m$ phases, the total input power $P_{1}$ becomes:

$P_{1}=m P=2 m U I_{m} \frac{\theta_{w}}{\tau_{r}}$

Denoting the efficiency as $\eta$, the total output power $P_{2}$ is written as:

$P_{2}=\eta P_{1}=2 m U I_{m} \frac{\theta_{w}}{\tau_{r}} \eta$ 
Substituting $\tau_{r}$ into (4), it yields:

$$
P_{2}=\frac{p_{r}}{\pi} m k_{e} E I_{m} \theta_{w} \eta
$$

where $k_{e}=\frac{U}{E}$, and $E$ is the phase back-emf due to the variation of PM flux linkage. This backemf can be expressed as:

$$
\begin{aligned}
E & =w \frac{d \phi_{m}}{d \theta} \omega_{r} \approx w \frac{\phi_{m}-\phi_{0}}{\theta_{w}} \omega_{r} \\
& =w \frac{\Delta \phi_{m}}{\theta_{w}} \omega_{r}
\end{aligned}
$$

where $w$ is the number of turns in series per phase, $\phi_{m}$ and $\phi_{0}$ are the PM flux in. Wb linked by one coil when the stator pole aligns and nonaligns with the rotor pole, respectively. In general, $\Delta \phi_{m}$ can further be expressed as:

$$
\begin{aligned}
\Delta \phi_{m} & =\phi_{m}-\phi_{0} \approx 0.9 \phi_{m} \\
& =0.9 k_{d} \alpha_{s} \tau_{s} l_{e} B_{\delta}=0.9 k_{d} \alpha_{s} \frac{i \tau D_{i}}{p_{s}} l_{e} B_{\delta}
\end{aligned}
$$

where $k_{d}$ is the PM flux leakage factor, $l_{e}$ is the stack length in $\mathrm{m}, B_{\delta}$ is the air-gap flux density in $\mathrm{T}, \tau_{s}=\pi D_{i} / p_{s}$ is the stator pole pitch in $\mathrm{m}$, $\alpha_{s}$ is the stator pole arc factor, $p_{s}$ is the stator pole number, and $D_{i}$ is the stator inner diameter in $\mathrm{m}$. Substituting (7) into (6), the back-emf can be written as:

$$
E=\frac{0.9 \pi k_{d} w \alpha_{s} D_{i} l_{e} B_{\delta}}{p_{s} \theta_{w}} \omega_{r}
$$

On the other hand, $I_{m}$ can be expressed as:

$I_{m}=k_{i} I_{m s}=k_{i} \pi D_{i} A / 2 m w$

where $A$ is the surface current density in $A / m$, $I_{m s}$ is the rms phase current in A, and $k_{i}=I_{m} / I_{m s s}$. Substituting (8) and (9) into (5) and adopting the general case that $\alpha_{s} \approx 0.5$, the output power of this motor can be derived as:

$$
P_{2}=\frac{0.9 \pi^{2}}{120} \frac{p_{r}}{p_{s}} k_{d} k_{e} k_{i} A B_{\delta} D_{i}^{2} l_{e} n_{s} \dot{\eta}
$$

From (10), it can be found that the total output power is directly proportional to the ratio of rotor poles to stator poles, $p_{r} / p_{s}$. Given $A$ and $B_{\delta}$, the larger the value of $p_{r} / p_{s}$ can certainly possess the higher the power density. Thus, the 8/6-pole motor can offer higher power density than the $6 / 4$-pole one by $12.5 \%$.

Moreover, the superior power density of the DSPM motor can be illustrated by comparing with the induction motor. According to the theory of induction motor, the corresponding output power is given by:

$P_{2 M M}=\frac{\sqrt{2} \pi^{2}}{120} k_{w} k_{e} A B_{m} D_{i}^{2} l_{e} n_{s} \eta \cos \varphi$

where $k_{w}$ is the winding factor, and $B_{m}$ is the maximum air-gap flux density. Making use of (10) and (11), the power density ratio of these two motors can be obtained as:

$\xi=\frac{P_{2}}{P_{2 M}}=\frac{0.9}{\sqrt{2}} \frac{p_{r}}{p_{s}} \frac{k_{d} k_{i} B_{\delta}}{k_{w} B_{m} \cos \varphi}$

Notice that $k_{d}$ of the DSPM motor is generally from 0.8 to 0.9 [5] which is about the same range as $\cos \varphi$ in the induction motor, whereas $B_{\delta}$ of the DSPM motor is almost the same as the tooth flux density (about $1.5 \mathrm{~T}$ ) which is about twice the $B_{m}$ in the induction motor. Hence, the power density ratio can be further simplified as:

$\xi=\frac{1.8}{\sqrt{2}} \frac{p_{r}}{p_{s}} \frac{k_{i}}{k_{w}}$

As shown in Fig. 2 , it is generally valid that $\theta_{w} \approx \tau_{r} / 3$ for the DSPM motor. Hence, $k_{i}$ can be calculated as follows:

$$
\begin{aligned}
I_{m m s} & =\sqrt{\frac{1}{T} \int_{0}^{T} i^{2} d t}=\sqrt{\frac{1}{\tau_{r}} 2 I_{m}^{2} \theta_{w}} \\
& =\sqrt{\frac{2}{\tau_{r}} \frac{1}{3} \tau_{r} I_{m}}=\sqrt{\frac{2}{3} I_{m}} \\
k_{i} & =\frac{I_{m}}{I_{m s}}=\sqrt{\frac{3}{2}}
\end{aligned}
$$

On the other hand, $k_{w}$ of the induction motor is generally about 0.96 or less. Substituting the numerical values of $k_{i}$ and $k_{w}$ into (13), it yields:

$\xi=\frac{1.8}{\sqrt{2}} \frac{6}{8} \frac{1}{0.96} \sqrt{\frac{3}{2}}^{\prime}=1.22$

It indicates that the proposed 8/6-pole DSPM motor possesses about $20 \%$ higher power density than that achieved by the induction motor when their stator surface current densities are the same. 


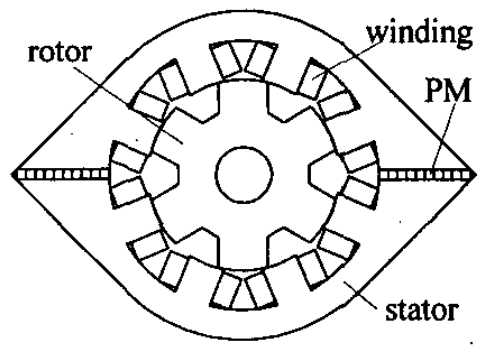

Fig. 1 Cross section of $8 / 6$-pole DSPM motor

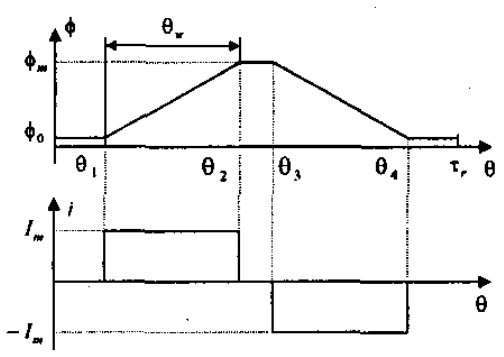

Fig. 2 Theoretical waveforms

\section{Dynamic Analysis}

The system matrix equation describing the proposed 4-phase 8/6-pole DSPM motor is expressed as:

$\bar{V}=\bar{R} \bar{I}+d \bar{\Psi} / d t$

where $\quad \bar{V}=\left[v_{1}, v_{2}, v_{3}, v_{4}\right]^{T}, \quad \bar{I}=\left[i_{1}, i_{2}, i_{3}, i_{4}\right]^{T}$

$\bar{R}=\operatorname{diag}\left[r_{1}, r_{2}, r_{3}, r_{4}\right]$, and $\bar{\Psi}=\bar{L} \bar{I}+\bar{\Psi}_{m}$. When both $\bar{L}$ and $\bar{\Psi}_{m}$ are considered to be spatially dependent only and independent of the stator current, it yields:

$$
\begin{aligned}
\frac{d \bar{\Psi}}{d t} & =\bar{L} \frac{d \bar{I}}{d t}+\frac{d \bar{L}}{d t} \vec{I}+\frac{d \Psi_{m}}{d t} \\
& =\bar{L} \frac{d \bar{I}}{d t}+\frac{d \bar{L}}{d \theta} \bar{I} \omega_{r}+\frac{d \bar{\Psi}_{m}}{d \theta} \omega_{r}
\end{aligned}
$$

Thus, the dynamic equation given by (17) can be rewritten as:

$$
\frac{d \bar{I}}{d t}=-\bar{L}^{-1}\left[\bar{R}+\frac{d \bar{L}}{d \theta} \omega_{r}\right] \bar{I}+\bar{L}^{-1}\left[\bar{V}-\frac{d \bar{\Psi}_{m}}{d \theta} \omega_{r}\right]
$$

By employing the co-energy method, the torque equation of the motor is obtained as:

$$
\begin{aligned}
T_{e} & =\frac{\partial W^{\prime}}{\partial \theta}=\frac{\partial}{\partial \theta}\left[\frac{1}{2} \bar{I}^{T} \bar{L} \bar{I}+\bar{\Psi}_{m}^{r} \bar{I}\right] \\
& =\frac{1}{2} \bar{I}^{T}\left(\frac{\partial}{\partial \theta} \bar{L}\right) \bar{I}+\left(\frac{\partial}{\partial \theta} \bar{\Psi}_{m}\right)^{r} \bar{I}=T_{r}+T_{m}
\end{aligned}
$$

in which $T_{r}=\frac{1}{2} \bar{I}^{T}\left(\frac{\partial}{\partial \theta} \bar{L}\right) \bar{I}$ represents the reluctance torque due to the variation of inductances, and $T_{m}=\left(\frac{\partial}{\partial \theta} \bar{\Psi}_{m}\right)^{T} \vec{I}$ is the reaction torque due to the interaction between the winding current and PM flux. Computer simulation can readily be performed by numerically solving the dynamic equation given by (19). Hence, the output torque can be simulated by using (20).

Besides using numerical techniques, an analytical solution of (19) is highly desirable which can provide the designer physical insight into the motor performance. By neglecting the mutual inductances $L_{i j}(i \neq j)$ of the motor, (19) can be decoupled among phases and the state equation for one phase is represented by:

$\frac{d i}{d t}=-\frac{r+\frac{d L}{d \theta} \omega_{r}}{L} i+\frac{U-\frac{d \psi_{m}}{d \theta} \omega_{r}}{L}$

where $r$ and $L$ are respectively the resistance and self-inductance of each phase winding. Taking $L$ as its average value in one stroke, the analytical solution of (21) can be obtained as:

$i=\frac{U-\frac{d \psi_{m}}{d \theta} \omega_{r}}{r+\frac{d L}{d \theta} \omega_{r}}\left(1-e^{-\frac{r+\frac{d L}{L} \omega_{r}}{L} t}\right)$

Hence, the steady-state phase current is given by:

$$
\dot{I}=\left(U-\frac{d \psi_{m}}{d \theta} \omega_{r}\right) /\left(r+\frac{d L}{d \theta} \omega_{r}\right)
$$

According to the operating principle of the DSPM motor, a positive current should be applied to the phase winding when the corresponding flux linkage is increasing, and vice versa. To keep a positive current at the first stroke and a negative current at the second stroke, the following condition is necessary:

$U-\frac{d \Psi_{m}}{d \theta} \omega_{r} \geq 0$

It illustrates that for a given supply voltage, there is a velocity limit $\omega_{r_{\max }}$ as represented by:

$\omega_{r \max }=\frac{U}{\frac{d \psi_{m}}{d \theta}}=\frac{U}{w \frac{d \phi_{m}}{d \theta}}=\frac{U}{w \frac{\Delta \phi_{m}}{\theta_{w}}}$ 
From (7), it yields:

$$
\Delta \phi_{m}=0.9 k_{d} \beta_{s} \frac{D_{i}}{2} l_{e} B_{\delta}=k B_{\delta} \beta_{s}
$$

where $k$ is a constant governed by the motor dimensions and $\beta_{s}$ is the stator pole arc in rad. For the general case that $\beta_{r}>\beta_{i}^{\prime}$ and $\beta_{s}=\theta_{w}$, (25) becomes:

$$
\omega_{r \max }=\frac{U}{w k B_{\delta} \beta_{s} / \theta_{w}}=\frac{U}{k w B_{\delta}}
$$

Since $B_{\delta}$ does not significantly; vary with the phase number while $w$ generally, decreases with the increase in phase number for a given structural size, (27) indicates that the 4-phase 8/6-pole DSPM motor can offer' a wider speed range than the 3-phase 6/4-pole one. This equation also reveals that there are two ways to extend the speed range of the DSPM motor: one is to weaken the magnetic field as described in [5]; another is to change the number of turns per phase. More detailed discussion on the extension of speed range by changing the number of turns of phase windings will be reported in another paper.

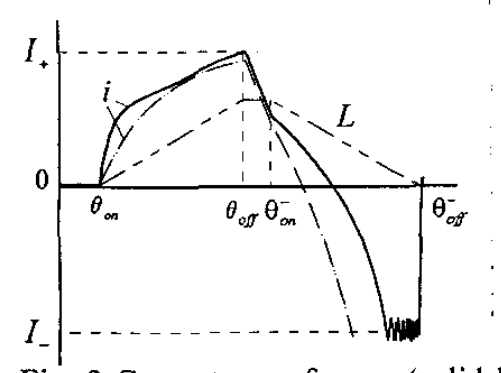

Fig. 3 Current waveforms (solid line: numerical simulation; dashed line: analytical' solution)

It should be noted that the analytical solution given by (22) is a simplified expression of the phase current, which aims to describe its tendency and profile. To illustrate its validity and discrepancy, the phase current obtained from (22) is plotted with the numerically simulated phase current from (19) as shown in Fig. 3. As expected, both the numerical and analytical results illustrate that the current waveform is asymmetric between the two strokes when the DSPM motor is operating above the base speed. In the positive stroke, the winding current initially rises very fast and then slows down with the increase in inductance. At high speeds, this current may not be able to reach its steady-state value before the winding is switched off at the rotor position angle $\theta_{o f f}$. Thus, both $\theta_{o n}$ and $\theta_{\text {off }}$ are possible control variables to shape the winding current. In the negative stroke, however, the current may rise rapidly to its steady-state value which can be explained by (23) with a negative $d L / d \theta$. Thus, the current regulation needs to be employed in the negative stroke even at high speeds. In practice, it may not be necessary to control $\theta_{o n}^{-}$and $\theta_{o f f}^{-}$though they are also control variables.

\section{Computer Simulation}

A 4-phase 8/6-pole DSPM motor as shown in Fig. 1 has been. designed to illustrate the aforementioned analysis. For a meaningful comparison, the motor is of the same major parameters, namely outer diameter, inner diameter and stack length of the stator, as a 750W induction motor. Firstly, finite element analysis of the proposed 8/6-pole DSPM motor is carried out, in which magnetic saturation has been taken into account. A typical field pattern is shown in Fig. 4. After this field analysis, the flux linkage and inductances can be deduced. Fig. 5 shows the characteristics in variation of the rotor position angle.

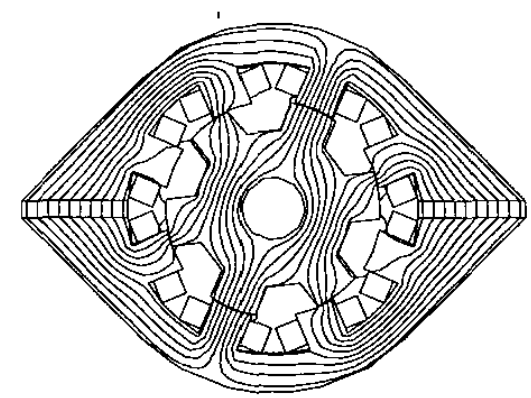

Fig. 4 Field pattern using finite element analysis

Based on the parameters resulting from the finite element analysis and the previously derived equations, computer simulation of the proposed motor performance is carried out. The motor voltage, current and various torque waveforms of the 8/6-pole DSPM motor at speeds below and above the base speed are shown in Figs. 6 and 7, respectively. At the low speed, the phase current is kept constant in each stroke by chopping. As a 
result, the reluctance torque has a near-zero average value which exhibits little contribution to the overall torque but causes some torque ripples. At the high speed, the phase current can no longer be maintained constant because of the excessive PM induced EMF. It should be noted that the uneven distribution of the phase current causes a significant amount of negative average reluctance torque, leading to reduce the constant power capability of the motor. This adverse effect can be minimized by properly controlling $\theta_{o n}$ and $\theta_{o f f}$.

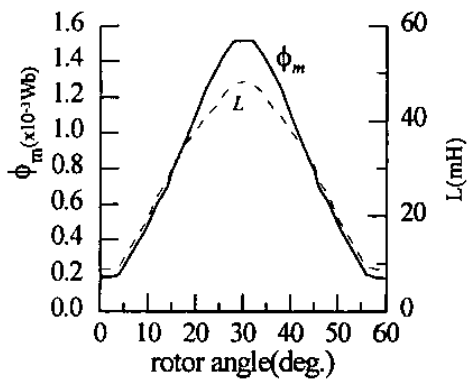

Fig. 5 Characteristics of PM flux and selfinductance
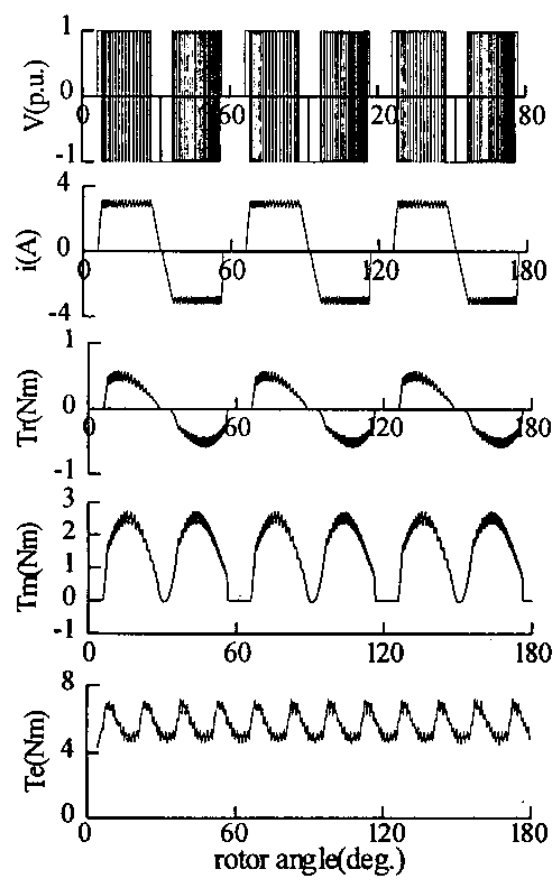

Fig. 6 Waveforms at $1000 \mathrm{rpm}\left(\theta_{o n}=6^{\circ}\right.$, $\theta_{\text {off }}=28^{\circ}$ )
Fig. 8 shows the overall torque and output power capabilities with respect to different combinations of $\theta_{\text {on }}$ and $\theta_{\text {off }}$ of the proposed 8/6-pole DSPM motor. It can be found that the maximum torque and power capabilities can be attained when $\theta_{o n}$ and $\theta_{\text {off }}$ are selected as $3^{\circ}$ and $25^{\circ}$, respectively. Hence, the output power capability of the proposed 8/6-pole DSPM motor operating at $1500 \mathrm{rpm}$ is $892 \mathrm{~W}$.
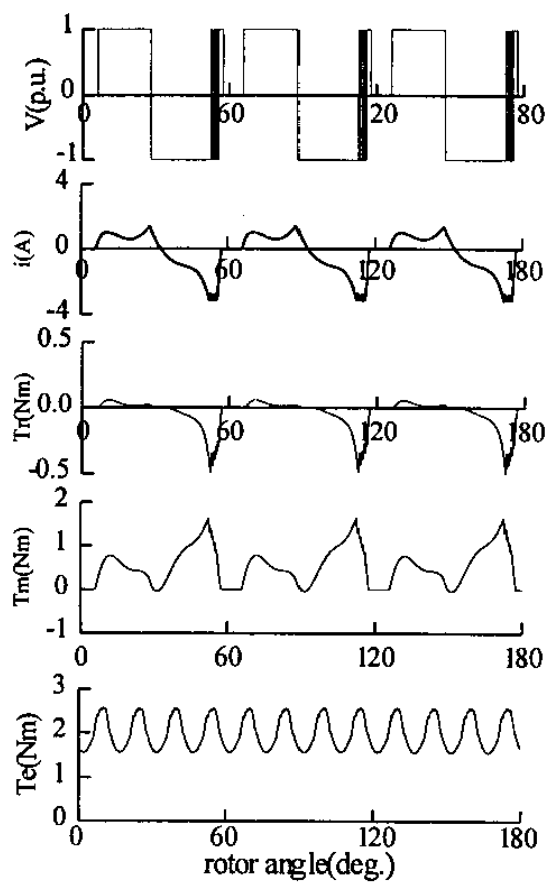

Fig. 7 Waveforms at $2500 \mathrm{rpm}\left(\theta_{o n}=6^{\circ}\right.$, $\theta_{\text {off }}=28^{\circ}$ )

\section{Comparisons of $8 / 6$ - and 6/4-Pole DSPM Motors}

Based on the same dimensions of the 8/6-pole DSPM motor, the design of the 6/4-pole one is also carried out. Provided that their surface current densities are the same, the output power capability of the 6/4-pole motor operating at $1500 \mathrm{rpm}$ is found to be $820 \mathrm{~W}$. Comparing with the $892 \mathrm{~W}$ offered by the $8 / 6$-pole one, the power density of the $6 / 4$-pole motor is $8 \%$ lower than that of 8/6-pole one. Moreover, as shown in Fig. 9 , it can been observed that the 8/6-pole motor can achieve higher output power and wider speed 
range while offering less phase current than the 6/4-pole one.

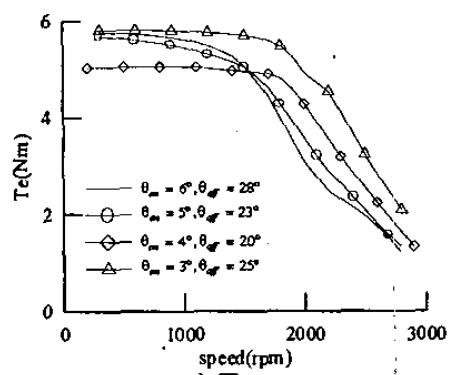

a) Torque

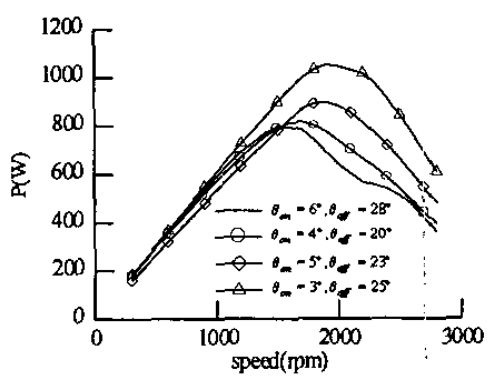

b) Power

Fig. 8 Torque and power capabilities

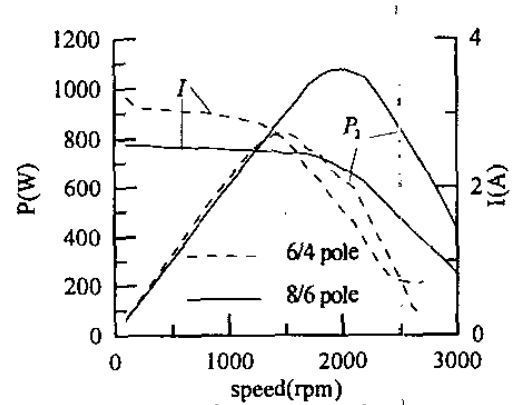

Fig. 9 Comparison of power and current

\section{Conclusions}

In this paper, the performance analysis of the newly proposed 8/6-pole DSPM niotor has been presented. Both steady-state and dynamic analyses of this motor have been given. The derived analytical solution can offer the definite advantage of physical insight for motor design, while the numerical simulation results can provide an accurate evaluation of the motor performance. Both analytical and numerical results have shown that the $8 / 6$-pole DSPM motor takes advantages over the 6/4-pole counterpart, namely higher power density, wider speed range, less torque ripple and lower current magnitude. These advantages can well outweigh its drawbacks on relatively more complex in structure and control. The prototypes are under construction and their experimental motion control will be reported in next papers.

\section{Acknowledgement}

This work was supported in part by the Committee on Research and Conference Grants of the University of Hong Kong, the Hong Kong Research Grants Council, and the National Natural Science Foundation of China (Project 59507001).

\section{References}

[1]C.C. Chan, Q. Jiang, Y.J. Zhan and K.T. Chau, "A high-performance switched reluctance drive for P-star EV project," EVS-13, Vol. II, 1996, pp. 78-83.

[2]C.C. Chan, K.T. Chau, J.Z. Jiang, W. Xia, M. Zhu and R. Zhang, "Novel permanent magnet motor drives for electric vehicles," IEEE Trans. Industrial Electronics, Vol. 43, No. 2, 1996, pp. 331-339.

[3]Y. Liao and T.A. Lipo, "A new doubly salient permanent magnet motor for adjustable speed drives," Electric Machines and Power Systems, Vol. 22, No. 1, 1993, pp. 259-270.

[4]Y. Liao, F. Liang and T.A. Lipo, "A novel permanent magnet motor with doubly salient structure," IEEE Trans. Industry Applications, Vol. 31, No. 5, 1995, pp. 10691078.

[5]Y. Li and T.A: Lipo, "A doubly salient permanent magnet motor capable of field weakening," IEEE PESC, 1995, pp. 565-571.

[6]F. Blaabjerg, L. Christensen, P.O. Rasmussen, L. Oestergaard; and P. Pedersen, "New advanced control methods for doubly salient permanent magnet motor," IEEE IAS, 1995, pp. 272-230.

[7]M.M. Radulescu, C. Martis and K. Biro, "A new electronically-commutated doublysalient permanent-magnet motor," IEE EMD, 1995, pp. 213-216.

[8]X. Luo, D. Qin and T.A. Lipo. "A novel two phase doubly salient permanent magnet motor," IEEE IAS, 1996, pp.808-815. 